\title{
DEFICIENCIES IN THE NUTATION MODEL FROM VLBI OBSERVATIONS
}

\author{
Y. F. XIA AND J. GAO \\ Department of Astronomy \\ Nanjing University \\ Nanjing, 210008 \\ P. R. China
}

\begin{abstract}
ABSTRAC'T. A VLBI series of celestial pole offsets has been used for deriving the correctious to the main terms of the IAU 1980 nutation series using the least squares solution and amplitude spectra method. The results are compared with current geophysical models. The discrepancies between observations and theory are largely due to deficiencies in the nutation models. A retrograde periodic variation (at a period of 420.0 days) has bcen revealed. The free mode will give us futher information on the core-mantle boundary.
\end{abstract}

\section{INTRODUCTION}

The International Earth Rotation Service (IERS) Standards recommends the use of the IAU 1980 nutation theory. It is based on the series of Kinoshita for the rigid Earth model and the normalized response function of Wahr (1981). With the continuing advance in very long baseline interferometry (VLBI) technique, the discrepancies between nutation observations and IAU 1980 nutation theroy are revealed in some of the larger amplitudes. They can not be explained in terms of uncertainties in the determinations.

VLBI observations are compared with nutation theory. It is an important means for checking the various earth models, and provide basis for improving nutation theory. The interior structure and properties of the Earth determine the eigenfrequencies and eigenfunctions of its normal modes. There are three dominant normal modes in Wahr's computations: the Eulerian nutation or Chandler wobble, the retrograde free core nutation (RFC!N) or nearly diurnal free wobble (NDFW) and the tilt-over mode (TOM). The cliange in the $R F C N$ resonance frequency affects the amplitude of the retrograde annual nutation. The RFCN frequency inferred from VLBI observations provides quantitative constraints for the dynamic ellipticity of the core (Gwinn et al., 1986). Recently, Mathews et al.(1991a,b) and Vries et al. (1991) considered the effect of the dynamics of the solid inner core and found a new nutational normal mode: the prograde free core nutation (PFCN) (Vries et al. called the mode the free inner core nutation (FICIN)). The existence of this normal mode could be responsible for part of the discrepancies between observations and theory. 


\section{VLBI NUTATION ANGLE DATA ANALYSIS}

The observations of $\delta \Delta \psi$ and $\delta \Delta \epsilon$ from the CALC: 7.1 solution of the Crustal Dynamics Project (CDP), as provided by Goddard Space Flight Center (CSFC), were used (Caprette et al., 1990). Some 848 VLBI experiments carried out between June 1979 and January 1991, each of about 24 hours duration, were included in this analysis. The formal errors are at the order of 0.8 to 3 mas in longitude and 0.3 to 1.3 mas in obliquity. The corrections to the nutation angles are added to the priori values of the nutation angles (IAU 1980 nutation values) used in the VLBI data analysis to form the total nutation angles.

A bias, rate and corrections to the 18.6 year periodic term in nutation for both $\delta \Delta \psi$ and $\delta \Delta \epsilon$ are first determined in the least squares analysis using the entire time span. The bias could be interpreted as the systematic differences in the definition of the equinox and equator. The linear rate is used to "absorb" the effects of any errors in the rate of precession and in the terms of long period in the nutation series.

The way we adopted for the correction of 3399.2 day term is the same as McCarthy et al. (1991). The coefficients of the 3399.2 day term were made equal to the rigid Earth correction of Kinoshita and Souchay (1990). Thus no correction of the 33399.2 day term is solved for.

Then, the observations are adjusted by the corrections found above and a solution is

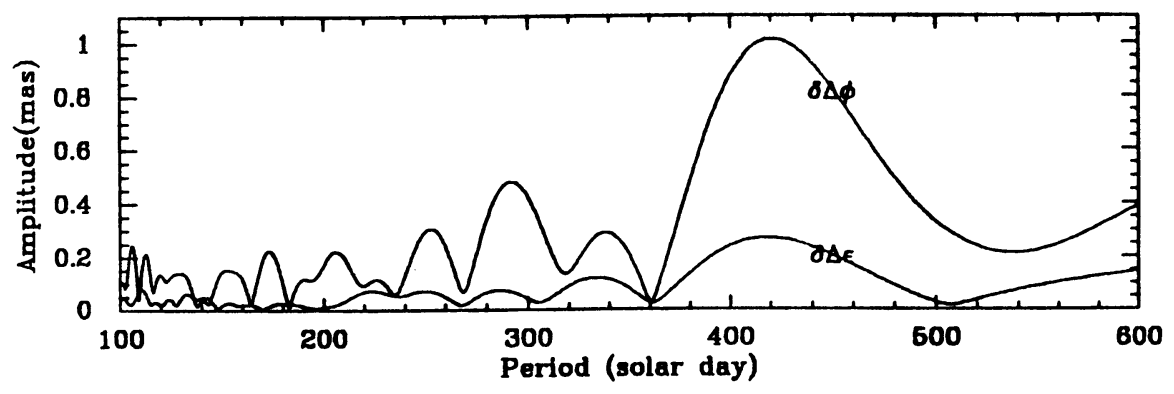

Figure 1. Smoothed amplitude spectra with the 420.0 day peak.

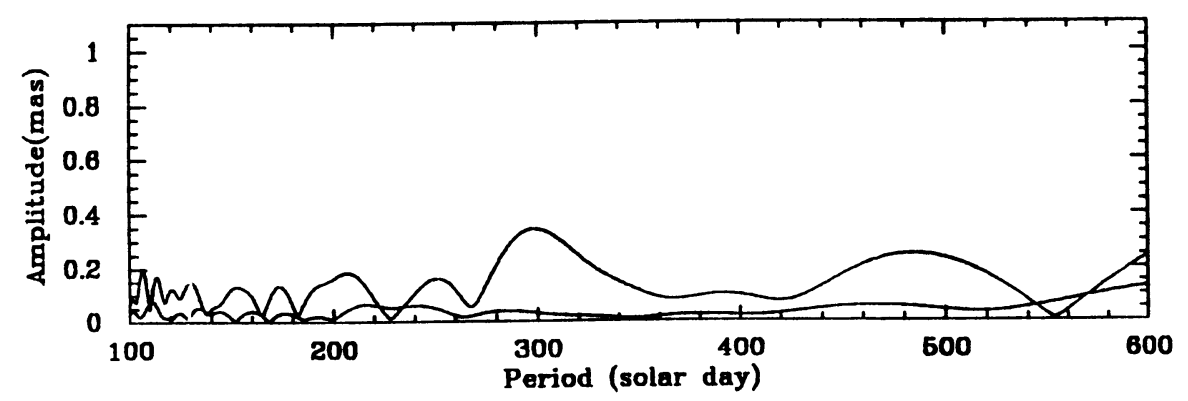

Figure 2. Smoothed amplitude spectra with all terms removed. 
made for the high-frequency terms (the seven largest nutation terms). The out-of-phase terms indicate the presence of dissipative processes in the Earth. To make the best possible use of the more accurate data when solving for the high-frequency terms, we have used only the 672 VLBI experiments which were made after MJD 45700.

The corrections to the high-frequency terms are used to adjust the observations again and amplitude spectra for the residual time series were used to determine the remaining power in the time series. Figure 1 shows the spectra. Peaks appear with average period 420.0 days in $\delta \Delta \psi$ and $\delta \Delta \epsilon$

Final estimates were obtained by iterating above steps for the long -period and shortperiod terms, including 420.0 day term. Once again the amplitude spectra for the residual time series were calculated. Figure 2 shows the spectra. No obvious peak appears.

TABLE 1. Comparison of the observed values with theoretical values. The units are arcsecond.

\begin{tabular}{rrrrr}
\hline$\Delta \psi$ & & & & \\
Period & IAU & Zhu and & ZMOA & $\begin{array}{r}\text { This } \\
\text { (days) }\end{array}$ \\
1980 & Groten & 1990 & Paper \\
\hline 6798.4 & -17.1996 & -17.2062 & -17.2063 & -17.2048 \\
3399.2 & 0.2062 & 0.2074 & 0.2075 & 0.2073 \\
365.3 & 0.1426 & 0.1474 & 0.1476 & 0.1478 \\
182.6 & -1.3187 & -1.3172 & -1.3172 & -1.3171 \\
121.7 & -0.0517 & -0.0518 & -0.0517 & -0.0518 \\
27.6 & 0.0712 & 0.0712 & 0.0711 & 0.0711 \\
13.7 & -0.2274 & -0.2282 & -0.2276 & -0.2280 \\
13.6 & -0.0386 & -0.0389 & -0.0387 & -0.0389 \\
9.1 & -0.0301 & -0.0302 & -0.0301 & -0.0303 \\
\hline$\Delta \epsilon$ & & & & \\
Period & IAU & Zhu and & ZMOA & This \\
(days) & 1980 & Groten & 1990 & Paper \\
\hline 6798.4 & 9.2025 & 9.2053 & 9.2051 & 9.2054 \\
3399.2 & -0.0895 & -0.0898 & -0.0898 & -0.0896 \\
365.3 & 0.0054 & 0.0072 & 0.0073 & 0.0074 \\
182.6 & 0.5736 & 0.5732 & 0.5731 & 0.5731 \\
121.7 & 0.0224 & 0.0225 & 0.0224 & 0.0225 \\
27.6 & -0.0007 & -0.0007 & -0.0007 & -0.0008 \\
13.7 & 0.0977 & 0.0981 & 0.0978 & 0.0979 \\
13.6 & 0.0200 & 0.0201 & 0.0201 & 0.0201 \\
9.1 & 0.0129 & 0.0129 & 0.0129 & 0.0129 \\
\hline & & & &
\end{tabular}

\section{RESULTS AND COMPARISONS}

The estimated corrections to the coefficient of IAU 1980 nutation series are calculated. The rms errors of the estimated correction are 1.52 and 0.52 mas for longitude and obliquity respectively except for the 18.6 year term, bias and rate terms, whose rms errors are estimated to be 4.43 and 1.62 mas. Table 1 compares the observed values with theoretical values from 
IAU 1980, Zhu et al. and ZMOA 1990 nutation series. Zhu et al. (1990) nutation series are based on the Zhu and Groten (1989) rigid -Earth nutation series and Wahr type normalized response function with modified coefficients. These coefficients were estimated from VLBI nutation angle data. ZMOA 1990 nutation series (Herring,1991) are the combination of the Zhu and Groten rigid-Earth nutation series, the response function developed by Mathews et al. (1991a,b), and the correction for ocean tides and anelasticity.

TABLE 2. Circular nutation amplitudes and corrections to the ZMOA 1990 and the IAU 1980 nutation series.

\begin{tabular}{rrrrrr}
\hline $\begin{array}{r}\text { Period } \\
\text { Solar Days }\end{array}$ & $\begin{array}{r}a_{r} \\
\text { mas }\end{array}$ & $\begin{array}{r}\delta a_{r} \\
\text { mas }\end{array}$ & $\begin{array}{r}a_{i} \\
\text { mas }\end{array}$ & $\begin{array}{r}\delta a_{i} \\
\text { mas }\end{array}$ & $\begin{array}{r}\delta a_{I A U} \\
\text { mas }\end{array}$ \\
\hline-9.13 & -0.44 & 0.01 & -0.01 & -0.01 & 0.02 \\
-13.63 & -2.32 & -0.02 & -0.03 & -0.03 & 0.00 \\
-13.66 & -3.61 & 0.03 & 0.06 & 0.06 & 0.01 \\
-27.55 & -13.75 & 0.06 & -0.05 & -0.03 & 0.06 \\
-121.75 & -0.93 & 0.01 & 0.03 & 0.03 & -0.02 \\
-182.62 & -24.58 & 0.07 & -0.03 & 0.02 & -0.05 \\
-365.26 & -33.10 & -0.10 & 0.39 & 0.46 & -2.04 \\
-3399.19 & 86.03 & -0.17 & 0.00 & 0.00 & 0.27 \\
-6798.38 & -8024.51 & 0.19 & 0.15 & -0.71 & -2.46 \\
6798.38 & -1180.84 & -0.43 & 1.86 & 1.97 & -0.39 \\
3399.19 & 3.57 & -0.03 & 0.00 & 0.00 & 0.33 \\
365.26 & 25.70 & -0.01 & 0.14 & 0.12 & 0.04 \\
182.62 & -548.51 & -0.01 & -0.46 & 0.05 & 0.56 \\
121.75 & -21.54 & -0.04 & -0.01 & 0.01 & -0.05 \\
27.55 & 14.52 & 0.04 & -0.03 & -0.04 & 0.00 \\
13.66 & -94.30 & -0.15 & -0.12 & -0.12 & -0.22 \\
13.63 & -17.79 & -0.09 & -0.04 & -0.04 & -0.11 \\
9.13 & -12.50 & -0.06 & -0.02 & -0.03 & -0.06 \\
& & & & & \\
-420.0 & 0.31 & & -0.07 & & \\
420.0 & -0.03 & & -0.13 & & \\
\hline
\end{tabular}

For geophysical studies the amplitudes of the circular nutations are more convenient to use because of the frequency dependence of the eigenmodes. The elliptical nutational motion in quasi-inertial space is shown as the combination of two circular motions in the rotating Earth system. In Table 2 we give the circular nutation amplitudes. Corrections to the ZMOA 1990 and the IAU 1980 nutation series are also given in this table. $\delta a_{r}$ and $\delta a_{i}$ are corrections to the in-phase and out-of-phase terms in the ZMOA 1990 nutation series. $\delta a_{I A U}$ is corrections to the IAU 1980 nutation series. IAU 1980 nutation series contains no out-of-phase terms. Amplitudes at the resonance frequency of the RFC'N and at the corresponding prograde frequency were also estimated. Table 3 lists the theoretical and VLBI observed values of the RFCNN resonance frequency derived by various authors. 


\section{DISCUSSION}

The errors at a level of a few mas were found in IAU 1980 nutation. The discrepancies can be interpreted as being due to the combined contribution of (1) imperfections of the rigidEarth model, (2) deficiencies in the nonrigid Earth nutation model used by Wahr, and (3) other geophysical reasons which have not been taken into consideration. Zhu et al. (1990) and Mathews et al.(1991a,b) considered these reasons and made much improvement. They give the new nutation series respectively. From the comparison of the observed values of this paper with the two new nutation values shown in table 1, it appears that observations agree with the geophysical theories at a level of mas.

TABLE 3. Theoretical and observed values of the RFCN frequency

\begin{tabular}{lll}
\hline Author & $\begin{array}{l}\text { NDFW Frequency } \\
\text { cpsd }\end{array}$ & $\begin{array}{l}\text { RFC'N Frequency } \\
\text { cpsd }\end{array}$ \\
\hline Theoretical values & & $-1 / 451.9$ \\
Guttenberg et al.(1961)* & -1.0022128 & $-1 / 472.9$ \\
Bullen et al.(1967)* & -1.0021155 & $-1 / 466.6$ \\
Wang (1972)* & -1.0021431 & $-1 / 460.5$ \\
Wahr 1066A (1981) & -1.0021714 & \\
Mathews et al.(1991a,b) & & $-1 / 458.4$ \\
(1) 1066A & -1.0021816 & $-1 / 457.0$ \\
(2) PREM & -1.0021882 & $-1 / 431.0$ \\
(3) Modified PREM & -1.0023203 & $-1 / 457$ \\
Vries et al. PREM (1991) & -1.0021882 & \\
& & $-1 / 432.7$ \\
Observed values & & $-1 / 433.2$ \\
Eubanks et al. (1985) & -1.0023111 & $-1 / 433.6$ \\
Herring et al.(1986) & -1.0023084 & $-1 / 430$ \\
Zhu et al.(1990) & -1.0023062 & $-1 / 418$ \\
Capitaine et al. (1991) & -1.0023256 & $-1 / 421.1$ \\
McCarthy et al. (1991) & -1.0023923 & \\
This Paper & -1.0023747 & \\
\hline
\end{tabular}

* From Herring et al. (1986)

From Table 2, the differences between the VLBI and the ZMOA 1990 estimates of the amplitudes are consistent with the rms errors of the VLBI estimates.

The freely excited RFC!N mode was detected from VLBI observation. The estimated amplitude of this mode given in Table 2 on the line corresponding to the period -420.0 days, is 0.32 mas, we also estimated the amplitude of a prograde term with the same period and obtained 0.13 mas.

Using the assumption of a hydrostatic Earth, the dynamic ellipticity of the core, $\epsilon_{f}=2.546 \times 10^{-3}$ was inferred from $(-1-1 / 460.5) \mathrm{cpsd}$. Mathews et al. adopted a "modifed PREM", and obtained $\omega_{R F C N}=-1 / 431.0 \mathrm{cpsd}$. The corresponding value of $e_{f}$ is found to be $2.672 \times 10^{-3}$. According to the VLBI $\omega_{R F C N}$ estimate of this paper, $\epsilon_{f}=2.726 \times 10^{-3}$. If the change of $\epsilon_{f}$ is attributed to an extra flattening of the core-mantle boudary relative to 
hydrostatic equilibrum, it is equivalent to an increase in the difference between the equatorial and polar radii of the core-mantle boundary. Thus, the discrepancies between observations and theory are largely due to deficiencies in the nutation models. The VLBI observational results for the RFCN resonance frequency are useful in constraining the dynamic ellipticity of the core. Vries et al.(1991) showed that this constraint is valid, even in the presence of a solid inner core, and the hydrostatic analytical result for RFCN resonance frequency is also valid in the nonhydrostatic case. The nonhydrostatic structure has no appreciable effect on RFC.N resonance frequency.

\section{REFERENCES}

Capitaine, N. and Caze, B. (1991), 'Deficiencies in the model for the celestial motion of the CEP as derived from a Goddard/VLBI series of pole offset from 1979 to 1989', in Proceedings of the IAU Colloquium 127, Reference Systems, edited by J. A. Hughes, C. A. Smith and G. H. Kaplan, USNO, Washington, D. C.., pp.222-223.

Caprette, D. S., Ma, C. and Ryan, J. W. (1990), Crustal Dynamics Project Data Analysis-1990, NASA Technical Memorandum 100765.

Eubanks, T. M., Steppe. J. A. and Sovers, O. J. (1985), 'An analysis and intercomparison of VLBI nutation estimates', in Proceedings of the International Conference, Earth Rotation and the 'Terrestrial Reference Frame, pp.326-340.

Giwim, C. R., Herring, T. A. and Shapiro, I. I. (1986), 'Geodesy by radio interferometry: Studies of the forced nutations of the Earth 2. Interpretation', J. geophys. Res., 91, 4755-4765.

Herring, T. A. (1991), 'The ZMOA-1990 nutation series', in Proceedings of the IAU Colloquium 127, Reference System, edited by J. A. Hughes, C. A. Smith and G. H. Kaplan, USNO, Washington, D. C., pp.157-166.

Herring, T. A., Gwinn, C. R. and Shapiro, I. I. (1986), 'Geodesy by radio interferometry: Studies of the forced nutations of the Earth 1. Data analysis', J. Geophys. Res., 91, 4745-4754.

Herring, T. A., Buffett, B. A., Mathews, P. M. and Shapiro, I. I. (1991), 'Forced nutations of the earth: Influence of inner core dynamics 3. Very Long Interferometry Data Analysis', J. Geophys. Res., 96, 8259-8273.

Kinoshita, H. and Souchay, J. (1990), 'The theory of the nutation for the rigid Earth model at the second order' Celest. Mech., 48, 187-256.

Mathews, P. M., Buffett, B. A., Herring, T. A. and Shapiro, I. I. (1991a), 'Forced nutations of the earth: Influence of inner core dynamics 1. Theory', J. Geophys. Res.,96, 8219-8242.

Mathews, P. M., Buffett, B. A., Herring, T. A. and Shapiro, I. I. (1991b), 'Forced nutations of the earth: Influence of inner core dynamics 2. Numerical Results and Comparisons', J. Cieophys. Res., 96, 8243-8257.

McCarthy, D. D. and Luzum, B. J. (1991), 'Observations of luni-solar and free core nutation', Astron. J., 102, 1889-1895.

Vries, D. and Wahr, J. M. (1991), 'The effects of the solid inner core and nonhydrostatic structure on the earth's forced nutations and earth tides', J. Geophys. Res., 96, 8275-8293.

Wahr, J. M. (1981), 'The forced nutations of an elliptical, rotating, elastic and oceanless earth', Geophys. J. R. Astron. Soc., 64, 705.

Zhu, S. Y. and Groten, E. (1989), 'Various aspects of numerical determination of nutation constants I. Improvement of rigid-earth nutation', Aston. J., 98, 1104-1111.

Zhu, S. Y., Groten, E. and Reigber, Ch. (1990), 'Various aspects of numerical determination of nutation constants. II. An improved nutation series for the deformable earth', Astron. J., 99, 1024-1044. 